\title{
THE TORUS LEMMA ON CALIBRATIONS, EXTENDED
}

\author{
FRANK MORGAN \\ (Communicated by Jonathan M. Rosenberg)
}

\begin{abstract}
The whole face $G(\varphi)$ of $m$-planes calibrated by a torus $m$-form $\varphi$ is determined by the torus face $G_{T}(\varphi)$. Indeed, $G(\varphi)$ results from applying a new closure operation to $G_{T}(\varphi)$.
\end{abstract}

\section{INTRODUCTION}

Over the past ten years the theory of calibrations has illuminated the occurrence and structure of singularities in $m$-dimensional area-minimizing surfaces. This note gives an extension of a much-used lemma on calibrations, the Torus Lemma (cf. §3). Our observations bear on recent work of D. Nance [N, e.g. Corollary 3.8] and M. Messaoudene [Me].

For surveys on calibrations see [H1], [M1], [M2]. For basic concepts and definitions see [M3, §1, $\S]$, [M4, Chapter 4], the original paper [HL], or the new text [H2].

\section{Definitions}

In addition to the standard dual Euclidean norms on the exterior algebra $\Lambda^{m} \mathbf{R}^{n}$ and its dual $\Lambda^{m} \mathbf{R}^{n *}$, there is another important dual pair of norms, called mass and comass. The comass $\|\varphi\|^{*}$ of a form $\varphi \in \Lambda^{m} \mathbf{R}^{n^{*}}$ is the maximum value of $\varphi$ on the Grassmannian $G\left(m, \mathbf{R}^{n}\right)$ of oriented unit $m$ planes through 0 in $\mathbf{R}^{n}$ :

$$
\|\varphi\|^{*}=\max \left\{\langle\xi, \varphi\rangle: \xi \in G\left(m, \mathbf{R}^{n}\right)\right\} .
$$

A form $\varphi$ normalized to have comass 1 is called a calibration. The face $G(\varphi)$ of a calibration $\varphi$ consists of its maximum points in the Grassmannian:

$$
G(\varphi)=\left\{\xi \in G\left(m, \mathbf{R}^{n}\right):\langle\xi, \varphi\rangle=1\right\} .
$$

Received by the editors October 5, 1988 and, in revised form, December 19, 1988.

1980 Mathematics Subject Classification (1985 Revision). Primary 49F10; Secondary 52A20, 49F20.

Key words and phrases. Torus lemma, torus calibration, face of Grassmannian, mass decomposition.

This work was partially supported by a National Science Foundation Grant. 
The mass is the dual norm on $m$-vectors $\xi$ in $\wedge^{m} \mathbf{R}^{n}$ :

$$
\begin{aligned}
\|\xi\| & =\max \left\{\langle\xi, \varphi\rangle:\|\varphi\|^{*}=1\right\} \\
& =\min \left\{\sum a_{j}: \xi=\sum a_{j} \xi_{j}, \xi_{j} \in G\left(m, \mathbf{R}^{n}\right), a_{j}>0\right\} .
\end{aligned}
$$

A calibration $\varphi$ for which the maximum of $\langle\xi, \varphi\rangle$ is attained is said to calibrate $\xi$. An expression $\xi=\sum a_{j} \xi_{j}$ for which the minimum is attained is called a mass decomposition for $\xi$. A calibration $\varphi$ calibrates $\xi=\sum a_{j} \xi_{j}$ if and only if all the $\xi_{j}$ lie in the face $G(\varphi)$.

In the case that $n=2 m$ and $\mathbf{R}^{n}=\left(\mathbf{R}^{2}\right)^{m}$ consider the $m$-dimensional torus

$$
T=\left(S^{1}\right)^{m}=\left(G\left(1, \mathbf{R}^{2}\right)\right)^{m} \subset G\left(m, \mathbf{R}^{2 m}\right) \subset \bigwedge^{m} \mathbf{R}^{2 m} .
$$

The elements of $T$ are called torus planes. Let $T_{s}$ denote the span of $T$ in $\Lambda^{m} \mathbf{R}^{2 m}$. The torus span $T_{s}$ can also be described as the tensor product $T_{s}=\bigotimes_{i=1}^{m} \wedge^{1} \mathbf{R}^{2}$. Elements of $T_{s}$ are called torus m-vectors. Similarly the elements of the dualspace $T_{s}^{*}$ are called torus forms. The intersection of the face $G(\varphi)$ of any calibration $\varphi$ with the torus $T$ is called the torus face $G_{T}(\varphi)$.

\section{THE TORUS LEMMA}

The Torus Lemma ([M5, Lemma 4], cf. [DHM, §4]) says that a torus calibration attains its maximum value of 1 on the torus. Equivalently,

$$
\left\{\varphi \in T_{s}^{*}: \max _{\xi \in T}\langle\xi, \varphi\rangle=1\right\}=\left\{\varphi \in T_{s}^{*}:\|\varphi\|^{*}=1\right\} .
$$

We observe a few useful consequences.

(1) The unit mass ball intersects the torus span in the convex hull of $T$.

(2) A torus $m$-vector is calibrated by a torus calibration [Me, 4.4.2].

(3) A torus $m$-vector has a mass decomposition in terms of torus $m$-planes. (This generalizes [Me, 5.2.8, 5.4.3].)

(4) Let $\varphi$ be a torus calibration. An $m$-plane $\xi$ belongs to $G(\varphi)$ if and only if its projection $P \xi$ onto the torus span $T_{s}$ is a convex combination of $G_{T}(\varphi)$.

Consequence (4) includes the new observation that the face $G(\varphi)$ of a torus form $\varphi$ is determined by the torus face $G_{T}(\varphi)$. This fact is applied by D. Nance [N, e.g. Corollary 3.8]. Theorem 6 will exhibit this fact in another way.

The consequences follow immediately from $\left({ }^{*}\right)$. For example, we will verify (4). Since $\varphi$ is a torus calibration, $\varphi(\xi)=\varphi(P \xi)$. Clearly if $P \xi$ is a convex combination of $G_{T}(\varphi)$, then $\varphi(\xi)=\varphi(P \xi)=1$, so that $\xi \in G(\varphi)$. On the other hand, suppose $\xi \in G(\varphi)$, so that $\varphi(P \xi)=\varphi(\xi)=1$. For any other torus calibration $\varphi^{\prime}, \varphi^{\prime}(P \xi)=\varphi^{\prime}(\xi) \leq 1$. It follows by elementary convex geometry from the characterization $\left(^{*}\right)$ of torus calibrations as

$$
\left\{\varphi \in T_{s}^{*}: \max _{\xi \in T}\langle\xi, \varphi\rangle=1\right\}
$$

that $P \xi$ is a convex combination of $G_{T}(\varphi)$. 


\section{Definitions}

We call $A \subset G\left(m, \mathbf{R}^{n}\right)$ a $\mathbf{C} P^{1}$ if for some orthonormal basis $e_{1}, \ldots, e_{n}$ for $\mathbf{R}^{n}$ and the complex structure $J e_{1}=e_{3}, J e_{2}=e_{4}$ on $\mathbf{R}^{4}=\operatorname{span}\left\{e_{1}, e_{2}, e_{3}, e_{4}\right\}$.

$$
A=\left\{\text { the complex lines in } \mathbf{R}^{4}\right\} \wedge e_{5} \wedge \cdots \wedge e_{m+2} .
$$

Let $B \subset G\left(m, \mathbf{R}^{n}\right)$. We define the $\mathbf{C} P^{1}$-closure $C(B)$ of $B$ as the smallest subset of $G\left(m, \mathbf{R}^{n}\right)$ containing $B$ such that whenever two points of a $\mathbf{C} P^{1}$ lie in $C(B)$, the whole $\mathbf{C} P^{1}$ lies in $C(B)$.

Proposition 5 ([HM, Corollary 4.7$])$. Let $G(\varphi)$ be a face of $G\left(m, \mathbf{R}^{n}\right)$. If two points of a $\mathbf{C} P^{1}$ lie in $G(\varphi)$, then the whole $\mathbf{C} P^{1}$ lies in $\mathbf{G}(\varphi)$.

Theorem 6. Let $\varphi \in \bigwedge^{m} \mathbf{R}^{2 m^{*}}$ be a torus calibration. Then the face $G(\varphi)$ is the $\mathbf{C} P^{1}$-closure of the torus face $G_{T}(\varphi)$ :

$$
G(\varphi)=C\left(T_{T}(\varphi)\right)
$$

Remarks. This theorem subsumes both the Torus Lemma, which just says that $G_{T}(\varphi) \neq \varnothing$, and our new observation that the entire face of a torus calibration is determined by its torus face (cf. §3).

Proof. By Proposition 5, $G(\varphi) \supset C\left(G_{T}(\varphi)\right)$. We prove the opposite inclusion by induction on $m$. The result is trivial for $m=1$. Suppose $\varphi \in$ $\otimes_{j=1}^{m+1}\left(\bigwedge^{1} R^{2^{*}}\right) \subset\left(\bigwedge^{1} R^{2^{*}}\right) \otimes\left(\bigwedge^{m} R^{2 m^{*}}\right)$. Let $\xi \in G(\varphi)$. It is not hard to show ([HL, Lemma II.7.5]) that there are orthonormal bases $e_{1}, e_{2}$ for $\mathbf{R}^{2}$ and $f_{1}, \ldots, f_{2 m}$ for $\mathbf{R}^{2 m}$ and angles $\theta_{1}, \theta_{2} \in[0, \pi / 2]$ such that $\xi$ takes the form

$$
\xi=\left(\cos \theta_{1} e_{1}+\sin \theta_{1} f_{1}\right) \wedge\left(\cos \theta_{2} e_{2}+\sin \theta_{2} f_{2}\right) \wedge f_{3} \wedge \cdots \wedge f_{m+1} .
$$

Since $\varphi \in \bigwedge^{1} \mathbf{R}^{2 *} \otimes \bigwedge^{m} \mathbf{R}^{2 m^{*}}$,

$$
\begin{aligned}
\varphi(\xi) & =a \cos _{1} \sin \theta_{2}+b \sin \theta_{1} \cos \theta_{2} \\
& \leq \sqrt{a^{2} \cos ^{2} \theta_{1}+b^{2} \sin ^{2} \theta_{1}} \leq \max \{|a|,|b|\} \leq 1,
\end{aligned}
$$

where

$$
\begin{aligned}
& a=\left\langle e_{1} \wedge f_{2} \wedge \cdots \wedge f_{m+1}, \varphi\right\rangle, \\
& b=\left\langle f_{1} \wedge e_{2} \wedge \cdots \wedge f_{m+1}, \varphi\right\rangle .
\end{aligned}
$$

Hence, equality holds. Unless $a=b=1$, it follows that $\left\{\theta_{1}, \theta_{2}\right\}=\{0, \pi / 2\}$ and $\xi$ has a factor $e_{1}$ or $e_{2}$, say $e_{1}$. Thus $\xi=e_{1} \wedge \zeta$, for some $\zeta \in G\left(m, \mathbf{R}^{2 m}\right)$. Since $\left.e_{1}\right\lrcorner \varphi \in \otimes_{j=1}^{m} \wedge^{1} \mathbf{R}^{2^{*}}$ and $\left.\left\langle\zeta, e_{1}\right\lrcorner \varphi\right\rangle= \pm\left\langle e_{1} \wedge \zeta, \varphi\right\rangle= \pm 1$, by induction $\pm \zeta$ lies in $\left.C\left(G_{T}\left(e_{1}\right\rfloor \varphi\right)\right)$. Consequently $\xi=e_{1} \wedge \zeta$ belongs to

$$
\left.\left. \pm e_{1} \wedge C\left(G_{T}\left(e_{1}\right\lrcorner \varphi\right)\right) \subset \pm C\left(e_{1} \wedge G_{T}\left(e_{1}\right\lrcorner \varphi\right)\right) \subset C\left(G_{T}(\varphi)\right)
$$

as desired. 
If on the other hand $a=b=1$, then $\theta_{2}=\pi / 2-\theta_{1}$. Also, $e_{1} \wedge f_{2} \wedge \cdots \wedge f_{m+1}$ and $f_{1} \wedge e_{2} \wedge \cdots \wedge f_{m+1}$ both belong to $G(\varphi)$. As in the previous case, by induction both belong to $C\left(G_{T}(\varphi)\right)$. In addition for the complex structure $J e_{1}=f_{2}, J f_{1}=e_{2}$, both belong to the $\mathbf{C} P^{1}$

$$
A=\left\{\text { complex lines in span }\left\{e_{1}, f_{2}, f_{1}, e_{2}\right\}\right\} \wedge f_{3} \wedge \cdots \wedge f_{m+1} .
$$

Since $\theta_{2}=\pi / 2-\theta_{1}, \xi$ also belongs to $A$. Therefore $\xi \in C\left(G_{T}(\varphi)\right)$, as desired.

\section{REFERENCES}

[DHM] J. Dadok, R. Harvey, and F. Morgan, Calibrations on $\mathbf{R}^{8}$, Trans. Amer. Math. Soc. 307 (1988), 1-40.

[H1] R. Harvey, Calibrated geometries, Proc. Int. Cong. Math., 1983.

[H2] __ Spinors and calibrations, manuscript.

[HL] R. Harvey and H. B. Lawson, Jr., Calibrated geometries, Acta Math. 148 (1982), 47-157.

[HM] R. Harvey and F. Morgan, The faces of the Grassmannian of 3-planes in $\mathbf{R}^{7}$, Inventiones Math. 83 (1986), 191-228.

[Me] M. Messaoudene, The unit mass ball of three-vectors in $\mathbf{R}^{6}$, Ph.D. Thesis, MIT, 1988.

[M1] F. Morgan, Area-minimizing surfaces, faces of Grassmannians, and calibrations, Amer. Math. Monthly 95 (1988), 813-822.

[M2] __ Calibrations and new singularities in area-minimizing surfaces: a survey, Proc. Conf. Problemes Variationnels, Paris, 1988, preprint.

[M3] $\_$, The exterior algebra $\Lambda^{k} \mathbf{R}^{n}$ and area minimization, Lin. Alg. and its Appl. 66 (1985), $1-28$.

[M4] _ Geometric measure theory: a beginner's guide, Academic Press, New York, 1988.

[M5] _ _ On the singular structure of three-dimensional area-minimizing surfaces, Trans. Amer. Math. Soc. 276 (1983), 137-143.

[N] D. Nance, A class of weakened special Lagrangian calibrations, Indiana U. Math. J., (to appear).

Department of Mathematics; Williams College, Williamstown, Massachusetts 01267 